Pacific Journal of Mathematic 


\title{
A SEPARABLY CLOSED RING WITH NONZERO TORSION PIC
}

ANDY R. MAGID

\begin{abstract}
We give an example of a ring and a rank one projective module over that ring such that the square of the module is free but the module does not become free over any separable extension of the ring.
\end{abstract}

Every ideal class in the ring of integers in a number field can be split by an unramified extension. Over a commutative ring which is an algebra over the rationals every torsion element of Pic of the ring is split by a separable extension [3]. These examples suggest the question: is the torsion part of Pic of a separably closed ring trivial? We will exhibit a ring which shows the answer is negative. The ring arises as a slight modification of an example of Swan [5].

For any commutative ring $k, k^{X}$ denotes the group of units of $k$, $\mathrm{Cl}(k)$ denotes the divisor class group if $k$ is a domain, and $Q u(k)$ denotes the group of quadratic extensions of $k$. We use $Z$ for the integers and $Q$ for the rationals.

Definition. For any commutative ring $k$, let $k\left(S^{1}\right)=k\left[X_{0}, X_{1}\right] /$ $\left(X_{0}^{2}+X_{1}^{2}-1\right)$. Let $t_{i}$ be the image of $X_{i}$ in $k\left(S^{1}\right) . k\left(S^{1}\right)$ is graded $\bmod 2$; let $k\left(P^{1}\right)$ be the even graded piece and $L(k)$ the odd.

LEMma 1. $L(k)$ is a projective $k\left(P^{1}\right)$-module of rank 1 whose tensor square is free.

Proof. It suffices to check the first assertion for $k=Z$. By the argument in [5, p. 271] $L(Z)$ is projective of rank 1. The multiplication in $k\left(S^{1}\right)$ defines a homomorphism

$$
L(k) \bigotimes_{k\left(P^{1}\right)} L(k) \rightarrow k\left(P^{1}\right)
$$

whose image contains $t_{0}^{2}+t_{1}^{2}=1$ and is thus an isomorphism.

We will show that $L(Z)$ cannot be split by a separable extension of $Z\left(P^{1}\right)$. We begin by collecting some facts about the rings involved.

LEMMA 2. (a) $Q(i)\left(P^{1}\right)=Q(i)\left[v, v^{-1}\right]$ where $v=\left(t_{0}+i t_{1}\right)^{2}$

(b) $Q\left(P^{1}\right)^{X}=Q^{X}$

(c) $Z / 2 Z\left(P^{1}\right)$ is a polynomial ring (in one variable) over $Z / 2 Z$

(d) $L(Z / 2 Z)$ is freely generated by $t_{0}+t_{1}$

Proof. Let $K=Q(i)$. Then $K\left(S^{1}\right)=K\left[u, u^{-1}\right]$ where $u=t_{0}+i t_{1}$. 
$K\left[v, v^{-1}\right]$ is contained in $K\left(P^{1}\right)$ and $K\left[u, u^{-1}\right]$ is separable of rank two over both rings. Thus $K\left[v, v^{-1}\right]=K\left(P^{1}\right)$; this gives (a). Let $g$ be the automorphism of $K\left(P^{1}\right)$ defined by $g(i)=-i\left(\right.$ so $\left.g(v)=v^{-1}\right)$. Then the ring of $g$-invariants is $Q\left(P^{1}\right)$. Since $K\left(P^{1}\right)^{X}=K^{X}\{v\}$, the only invariant units are in $Q^{X}$, whence (b). For (c), we observe that $Z$ / $2 Z\left[X_{0}, a\right]$ where $a^{2}=1$ is isomorphic to $Z / 2 Z\left(S^{1}\right)$ when $X_{0}$ is sent to $t_{0}$ and $a$ to $t_{0}+t_{1}$. This isomorphism is graded and under it, $Z / 2 Z\left(P^{1}\right)$ corresponds to $Z / 2 Z\left[X_{0}, a\right]$. For (d), let $f_{i}=t_{i}\left(t_{0}+t_{1}\right)$. Then $f_{i}\left(t_{0}+\right.$ $\left.t_{1}\right)=t_{i}$, so $t_{0}+t_{1}$ generates $L(Z / 2 Z)$.

We remark that (c) implies that $Z / 2 Z\left(P^{1}\right)^{X}=\{1\}$ and (b) implies that $Z\left(P^{1}\right)^{X}=\{ \pm 1\}$.

Proposition 3. $Z\left(P^{1}\right)$ is a normal domain with Pic $\left(Z\left(P^{1}\right)\right)=\{1$, $L(Z)\}$.

Proof. We begin by establishing analogous assertions for $Q\left(P^{1}\right)$. Let $K, v, g$ be as in the proof of Lemma 2 and let $G=\{1, g\}$. Then $K\left(P^{1}\right)$ is a Galois extension of $Q\left(P^{1}\right)$ with group $G$. Let $U=X\left(P^{1}\right)^{X}$. Since by Lemma 2 (a) $K\left(P^{1}\right)$ is a $U F D, Q\left(P^{1}\right)$ is normal and there is an isomorphism $\mathrm{Cl}\left(Q\left(P^{1}\right)\right) \rightarrow H^{1}(G, U)$ [4, p. 55]. To compute the latter group, we use the exact sequence of $G$-modules

$$
1 \longrightarrow V \longrightarrow U \longrightarrow K^{X} \longrightarrow 1
$$

where $V$ is the subgroup generated by $v$. Since by Lemma 2 (b) $U^{G}=$ $Q^{X}=\left(K^{X}\right)^{G}$ and since $H^{1}\left(G, K^{X}\right)=1$ by Hilbert's Theorem 90, the cohomology sequence of the above sequence shows that $H^{1}(G, U)$ and $H^{1}(G, V)$ are isomorphic. Since $V$ is $G$-isomorphic with $Z$ with $g$ acting by multiplication by -1 , we see that $H^{1}(G, V)$ (and hence $\left.\mathrm{Cl}\left(Q\left(P^{1}\right)\right)\right)$ is of order two. Now let $S$ be the multiplicative set in $Z\left(P^{1}\right)$ generated by the integer primes. The integer primes remain prime in $Z\left(P^{1}\right)$ (for the odd primes this is trivial and for two it follows from Lemma 2 (c)). Since $S^{-1} Z\left(P^{1}\right)=Q\left(P^{1}\right)$ it follows from [4, p. 21] that $Z\left(P^{1}\right)$ is a normal domain with $\mathrm{Cl}\left(Z\left(P^{1}\right)\right)=\mathrm{Cl}\left(Q\left(P^{1}\right)\right)$. By [5, Thm. 4, p. 271] $L(Z)$ cannot be generated by a single element. It follows that $\{1, L(Z)\}=\operatorname{Pic}\left(Z\left(P^{1}\right)\right)=\mathrm{Cl}\left(Z\left(P^{1}\right)\right)$.

Next, we show that every connected Galois extension of $Z\left(P^{1}\right)$ is abelian.

LEMmA 4. Let $K$ be an algebraically closed field of characteristic zero. Then every connected Galois extension of $K\left[X, X^{-1}\right]$ is cyclic.

Proof. We may assume $K$ is the complex numbers. Let $T$ be a connected Galois extension of $K\left[X, X^{-1}\right]$ and let $E$ be the quotient field of T. Let $M$ and $P$ be the Riemann surfaces of $E$ and $K(X)$ 
respectively ( $P$ is just the Riemann sphere, of course). The inclusion of $K(X)$ in $E$ displays $M$ as a local branched covering of $P$ ramified only above 0 and $\infty$. The branching order formula [3, Cor. 3, p. 225] shows that $M$ has genus zero and only one branch point over each of 0 and $\infty$. It follows that the covering map is the $n^{\text {th }}$ power map, where $n=\left[T: K\left[X, X^{-1}\right]\right]$ and hence that $T=K\left[X, X^{-1}\right](\sqrt[n]{X})$. Then the Galois group of $T$ is cyclic of order $n$.

Proposition 5. Every connected Galois extension of $Z\left(P^{1}\right)$ is cyclic.

Proof. Let $K$ be the algebraic closure of $Q$ and $R$ the ring of all algebraic integers. Let $T$ be a connected Galois extension of $Z\left(P^{1}\right)$ with group $G$. Then $T \otimes_{z} R$ is a Galois extension of $R\left(P^{1}\right)$, and hence is a product of copies of a connected Galois extension $T_{0}$ of $R\left(P^{1}\right)$. The Galois group $H$ of $T_{0}$ is a subgroup of $G . T_{0} \otimes_{R} K$ is a connected Galois extension of $K\left(S^{1}\right)=K\left[v, v^{-1}\right]$ and hence by Lemma $4 H$ is cyclic. Choose a homomorphism (necessarily an injection) of $T$ into $T_{0}$. Then $T^{H}$ is a separable $Z\left(P^{1}\right)$-subalgebra of $T_{0}^{H}=R\left(P^{1}\right)$. Thus $T^{H}$ is contained in $S\left(P^{1}\right)$ where $S$ is the ring of integers in some finite extension of $Q$. Since $S\left(P^{1}\right)$ is $Z\left(P^{1}\right)$-projective and $T^{H}$ is $Z\left(P^{1}\right)$-separable, $T^{H}$ is a $T^{H}$-summand of $S\left(P^{1}\right)$. Let $f$ denote the evaluation $Z\left(S^{1}\right) \rightarrow Z$ (and also its restriction to $Z\left(P^{1}\right)$ ) at the point $(1,0)$. Then $T^{H} \otimes_{f} Z$ is a separable $Z$-subalgebra of $S\left(P^{1}\right) \otimes_{f} Z=S$. It follows that $T^{H} \otimes_{f} Z=Z$ and hence $Z\left(P^{1}\right)=T^{H}$.

THEOREM 6. $L(Z)$ cannot be split by a separable extension of $Z\left(P^{1}\right)$.

Proof. It will suffice to prove the theorem for connected Galois extensions; let $T$ be such an extension with group $G$. If $T$ splits $L(Z)$ then by Lemma $3 \operatorname{Pic}\left(Z\left(P^{1}\right)\right)=H^{1}\left(G, T^{X}\right)$. It follows that $G$ has even order. Since by Proposition $5 G$ is cyclic, it will suffice to show that $Z\left(P^{1}\right)$ has no connected quadratic extensions. Since for any normal domain $k$ with quotient field $L$ the map $Q u(k) \rightarrow Q u(L)$ is injective, $Q u\left(Z\left(P^{1}\right)\right)$ is contained in $Q u\left(Q\left(P^{1}\right)\right)$. To compute this latter group, we use the exact sequence of [1, p. 129] valid for any ring $k$ containing $1 / 2$ :

$$
1 \longrightarrow k^{x} /\left(K^{X}\right)^{2} \longrightarrow Q u(k) \longrightarrow 2-\operatorname{Pic}(k) \longrightarrow 1
$$

where the first map sends $a$ to $k[X] /\left(X^{2}-a\right)$, the second sends $T$ to $T / k$ and the fourth group in the sequence is the two-torsion part of Pic. Let $T$ be a quadratic extension of $Z\left(P^{1}\right)$ and let $I=T / Z\left(P^{1}\right)$. Let $T_{0}=T \otimes_{z} Q$ and let $I_{0}=T_{0} / Q\left(P^{1}\right)$. If $I=1$ and thus also $I_{0}=$ 1 , the above exact sequence shows that $T_{0}=Q(\sqrt{a})\left(P^{1}\right)$ for some $a$ 
in $Q$, since by Lemma 2 (b) $Q\left(P^{1}\right)^{X}=Q^{x}$. Let $f: Z\left(P^{1}\right) \rightarrow Z$ and $h$ : $Q\left(P^{1}\right) \rightarrow Q$ be induced by evaluation at $(1,0)$. Then $T \otimes_{f} Z \otimes_{z} Q=$ $T_{0} \otimes_{h} Q$, since the first is $Q \times Q$ and the second $Q(\sqrt{a}), a$ is in $Q$ and $T_{0}$ and therefore $T$ must be the trivial extension. To treat the case $I=L(Z)$ we use the following exact sequence, which is part of a sequence due to Small [6]: for any ring $k$,

$$
Q u(k) \longrightarrow \operatorname{Pic}_{(2)}(k) \longrightarrow U^{\prime}(k)
$$

where the middle group is those two-torsion elements of Pic $(k)$ which become free over $k / 2 k$ and the end group is $(k / 4 k)^{X}$ modulo the subgroup generated by the squares and the image of $k^{x}$; the first map sends $T$ to $T / k$ and the second sends $I$ to the class of $g(m, m)$ where $g: I \bigotimes_{k} I \rightarrow I$ is an isomorphism and $m$ is in $I$ with the image of $m$ becoming a basis for $I / 2 I$. By Lemma 2 and the remarks following we see that $U^{\prime}\left(Z\left(P^{1}\right)\right)=Z / 4 Z\left(P^{1}\right)^{X} /\{ \pm 1\}$, and by Lemma $2 d$ and Proposition 3 we see that $\operatorname{Pic}_{(2)}\left(Z\left(P^{1}\right)\right)=\{1, L(Z)\}$. To compute the image of $L(Z)$ in $U^{\prime}\left(Z\left(P^{1}\right)\right.$ ) we choose for $g$ the multiplication map (Lemma 1 ) and for $m$ the element $t_{0}+t_{1}$ (Lemma $\left.2(\mathrm{~d})\right)$. Then $g(m, m)=1+$ $2 t_{0} t_{1}$ has non-trivial image in $Z / 4 Z\left(P^{i}\right)$. Thus the case $I=L(Z)$ does not occur.

We conclude with some remarks: one can define $k\left(P^{n}\right)$ and $k\left(S^{n}\right)$ in a similar manner for $n$ larger than 1 . The arguments given here can be extended to cover these rings, except for Lemma 4. Presumably the analogue of Theorem 6 remains valid, however.

\section{REFERENCES}

1. H. Bass, Lectures on Topics in Algebraic K-Theory, Tata Institute of Fundamental Research, Bombay, 1967

2. L. Childs, Abelian Galois extensions of rings containing roots of unity, Illinois J. Math., 15 (1971).

3. R. Gunning, Lectures on Riemann Surfaces, Princeton University Press, Princeton, 1966

4. P. Samuel, Lectures on Unique Factorization Domains, Tata Institute of Fundamental Research, Bombay, 1964.

5. R. Swan, Vector bundles and projective modules, Trans. Amer. Math. Soc., 105 (1962)

6. C. Small, The group of quadratic extensions (to appear)

Received June 11, 1971 and in revised form December 9, 1971.

Columbia University 


\section{PACIFIC JOURNAL OF MATHEMATICS}

\section{EDITORS}

\section{H. SAMELSON}

Stanford University

Stanford, California 94305

\section{R. HOBBY}

University of Washington

Seattle, Washington 98105

\section{J. DugundjI}

Department of Mathematics University of Southern California Los Angeles, California 90007

RICHARD ARENS

University of California

Los Angeles, California 90024

\section{ASSOCIATE EDITORS}
E. F. BECKENBACH
B. H. NeumanN
F. WOLF
K. YOSHIDA

\section{SUPPORTING INSTITUTIONS}

\author{
UNIVERSITY OF BRITISH COLUMBIA \\ CALIFORNIA INSTITUTE OF TECHNOLOGY \\ UNIVERSITY OF CALIFORNIA \\ MONTANA STATE UNIVERSITY \\ UNIVERSITY OF NEVADA \\ NEW MEXICO STATE UNIVERSITY \\ OREGON STATE UNIVERSITY \\ UNIVERSITY OF OREGON \\ OSAKA UNIVERSITY
}

\author{
UNIVERSITY OF SOUTHERN CALIFORNIA \\ STANFORD UNIVERSITY \\ UNIVERSITY OF TOKYO \\ UNIVERSITY OF UTAH \\ WASHINGTON STATE UNIVERSITY \\ UNIVERSITY OF WASHINGTON \\ AMERICAN MATHEMATICAL SOCIETY \\ NAVAL WEAPONS CENTER
}

The Supporting Institutions listed above contribute to the cost of publication of this Journal, but they are not owners or publishers and have no responsibility for its content or policies.

Mathematical papers intended for publication in the Pacific Journal of Mathematics should be in typed form or offset-reproduced, (not dittoed), double spaced with large margins. Underline Greek letters in red, German in green, and script in blue. The first paragraph or two must be capable of being used separately as a synopsis of the entire paper. The editorial "we" must not be used in the synopsis, and items of the bibliography should not be cited there unless absolutely necessary, in which case they must be identified by author and Journal, rather than by item number. Manuscripts, in duplicate if possible, may be sent to any one of the four editors. Please classify according to the scheme of Math. Rev. Index to Vol. 39. All other communications to the editors should be addressed to the managing editor, Richard Arens, University of California, Los Angeles, California, 90024.

50 reprints are provided free for each article; additional copies may be obtained at cost in multiples of 50 .

The Pacific Journal of Mathematics is published monthly. Effective with Volume 16 the price per volume (3 numbers) is $\$ 8.00$; single issues, $\$ 3.00$. Special price for current issues to individual faculty members of supporting institutions and to individual members of the American Mathematical Society: $\$ 4.00$ per volume; single issues $\$ 1.50$. Back numbers are available.

Subscriptions, orders for back numbers, and changes of address should be sent to Pacific Journal of Mathematics, 103 Highland Boulevard, Berkeley, California, 94708.

PUBLISHED BY PACIFIC JOURNAL OF MATHEMATICS, A NON-PROFIT CORPORATION

Printed at Kokusai Bunken Insatsusha (International Academic Printing Co., Ltd.), 270, 3-chome Totsuka-cho, Shinjuku-ku, Tokyo 160, Japan. 


\section{Pacific Journal of Mathematics}

\section{Vol. 42, No. $3 \quad$ March, 1972}

Catherine Bandle, Extensions of an inequality by Pólya and Schiffer for vibrating membranes ................................ 543

S. J. Bernau, Topologies on structure spaces of lattice groups.......... 557

Woodrow Wilson Bledsoe and Charles Edward Wilks, On Borel product measures .......................................

Eggert Briem and Murali Rao, Normpreserving extensions in subspaces of

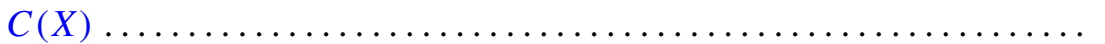

Alan Seymour Cover, Generalized continuation.................. 589

Larry Jean Cummings, Transformations of symmetric tensors .......... 603

Peter Michael Curran, Cohomology of finitely presented groups .......... 615

James B. Derr and N. P. Mukherjee, Generalized quasicenter and

hyperquasicenter of a finite group ...................... 621

Erik Maurice Ellentuck, Universal cosimple isols .................. 629

Benny Dan Evans, Boundary respecting maps of 3-mainfolds .......... 639

David F. Fraser, A probabilistic method for the rate of convergence to the

Dirichlet problem .................................. 657

Raymond Taylor Hoobler, Cohomology in the finite topology and Brauer

groups ..................................... 667

Louis Roberts Hunt, Locally holomorphic sets and the Levi form ........ 681

B. T. Y. Kwee, On absolute de la Vallée Poussin summability............ 689

Gérard Lallement, On nilpotency and residual finiteness in semigroups .... 693

George Edward Lang, Evaluation subgroups of factor spaces........... 701

Andy R. Magid, A separably closed ring with nonzero torsion pic ....... 711

Billy E. Rhoades, Commutants of some Hausdorff matrices ............. 715

Maxwell Alexander Rosenlicht, Canonical forms for local derivations . . . . 721

Cedric Felix Schubert, On a conjecture of L. B. Page ................ 733

Reinhard Schultz, Composition constructions on diffeomorphisms of $S^{p} \times S^{q}$

J. P. Singhal and H. M. (Hari Mohan) Srivastava, A class of bilateral generating functions for certain classical polynomials ....

Richard Alan Slocum, Using brick partitionings to establish conditions which insure that a Peano continuum is a 2-cell, a 2-sphere or an annulus...

James F. Smith, The p-classes of an $H^{*}$-algebra ...

Jack Williamson, Meromorphic functions with negative zeros and positive

poles and a theorem of Teichmuller ................. 